\title{
The Hunger Games as the Key to Happily Ever After?
}

\author{
Jacques A. Deere ${ }^{1, *}$, Aziz Aboobaker ${ }^{1}$ and Roberto Salguero-Gómez 1,2,3 \\ 1 Department of Zoology, 11a Mansfield Rd, University of Oxford, Oxford, OX1 3SZ, United Kingdom; \\ aziz.aboobaker@zoo.ox.ac.uk (A.A.); rob.salguero@zoo.ox.ac.uk (R.S.-G.) \\ 2 Centre for Biodiversity and Conservation Science, University of Queensland, St Lucia 4071QLD, Australia. \\ 3 Evolutionary Demography Laboratory, Max Plank Institute for Demographic Research, Rostock 18057, \\ Germany. \\ * Correspondence: jacques.deere@zoo.ox.ac.uk
}

\begin{abstract}
The world's human population is reaching record longevities. Consequently, societies are experiencing the tangible impacts of prolonged longevity, such as increased retirement age. A major hypothesised influence on ageing patterns is resource availability and calorie restriction, considered by many to extend longevity in any organism. Here, we highlight challenges facing the field of calorie restriction research as it pertains to ageing and how more realistic environments can impact the role calorie restriction plays in longevity of species. We reviewed 120 peer-reviewed published studies to quantify calorie restriction effects on longevity. We show that calorie restriction research does not always have positive effects on ageing with $27 \%$ of studies having no, negative or neutral effects. Additionally, research is biased towards short-lived species and lacks realism. We argue that only by taking a more realistic approach can the impacts of calorie restriction on longevity under climate change be understood. We conclude by discussing Planarians and Hydra as model species that allow for future research to have a better understanding of calorie restriction effects on longlived species, while incorporating climate change impacts. Steering future calorie restriction research towards integrating interaction effects across a broader range of species will begin addressing the challenges of calorie restriction research. Crucial insights from future research can contribute to the fundamental and translational understanding of human senescence.
\end{abstract}

Keywords: calorie restriction; longevity; environmental fluctuation; senescence

\section{Main}

Senescence is at the forefront of social, economic, and biological research ${ }^{1-4}$. This biological phenomenon is characterised by the physiological decline of an organism's vitality with age after reaching maturity, which ultimately reduces reproductive output and increases mortality risk. Exploring the implications of senescence is urgent because the world's population aged 65 and above is projected to increase from the current $12 \%$ to $16 \%$ by 2050 , doubling the old-age dependency ratio ${ }^{5}$. Indeed, some human societies are reaching record longevities, including Japan and Sweden, where the number of women aged 100 and above has increased over six-fold in only 25 years ${ }^{6}$. Human societies are already experiencing the tangible impacts of prolonged longevity, such as increasing age at retirement and economic consequent policies seeking to increase employment among people in their late 50's and early 60's6,7. Perhaps less widely appreciated is the fact that our society depends directly on the productivity accrued throughout the longevity of non-human species, via crucial ecosystem services such as carbon sequestration, which depends on the vitality and survival of forest trees $^{8}$, or crop production, which is sustained via reproduction ${ }^{9}$. Thus, beyond focusing only on humans, investigating why some species senesce but others do not ${ }^{2}$ will ultimately provide a fundamental and translational framework of understanding of senescence in humans ${ }^{10}$ and across the whole Tree of Life that is currently lacking2. Out of the over 300 existing theories on the evolution of senescence ${ }^{11}$, resource availability has been suggested as a major influence on ageing patterns. This 
idea was first proposed by Aristotle ${ }^{12}$ and is currently formalised under the umbrella of Calorie Restriction $(C R)$ theory.

In this Perspective, we focus on the impacts of calorie restriction on longevity. We discuss the current state of CR research and highlight several of its challenges, including lack of realism and limited taxonomic breath in experimental approaches. Throughout the Perspective we explore how interaction effects can impact longevity and potentially undermine the findings of published $\mathrm{CR}$ studies, particularly in light of global climate change. We conclude by exploring potential model species that can be used to incorporate $\mathrm{CR}$ and interaction effects to address the impact of more realistic resource quantity/quality conditions in fluctuating environments, as expected from climate change projections.

\section{Calorie restriction}

CR theory predicts that the onset of senescence is delayed and life expectancy prolonged due to the ultimate effects of restricted food intake without malnutrition ${ }^{13}$. The benefits of CR may be mediated at the molecular and cellular level by lowering molecular oxidative damage ${ }^{14}$ and reducing free radical-induced cellular damage ${ }^{15}$. Benefits of CR can also be mediated by activating pathways that lead to renewal of older/low-functioning cellular components, including autophagy through the modulation of hormonal signals that switch metabolic pathways ${ }^{16}$. Furthermore, CR may result in some species in behavioural changes that can expand lifespan, such as a change in activity levels to a state of torpor under $\mathrm{CR}^{17}$. The positive effects of $\mathrm{CR}$ was first observed in 1935 in a study on rats by McCay et al..$^{18}$ and has since been reported in several species, ranging from yeast, to invertebrates, and other mammals ${ }^{13,19-22}$. However, several challenges exist in CR research: (i) the effects of $C R$ are seemingly inconsistent across species, with some controversy over its positive or negative effects on organismal performance ${ }^{23}$ (Fig. 1); (ii) the ongoing variation in protocols and limitations of studies confounds the interpretation of the outcomes of CR research within and across species; (iii) $C R$ studies have been conducted mostly under constant laboratory conditions; and (iv) the range of species studied is still rather limited to infer its general effects. Together, these challenges limit our ability to unequivocally test predictions of CR theory. 


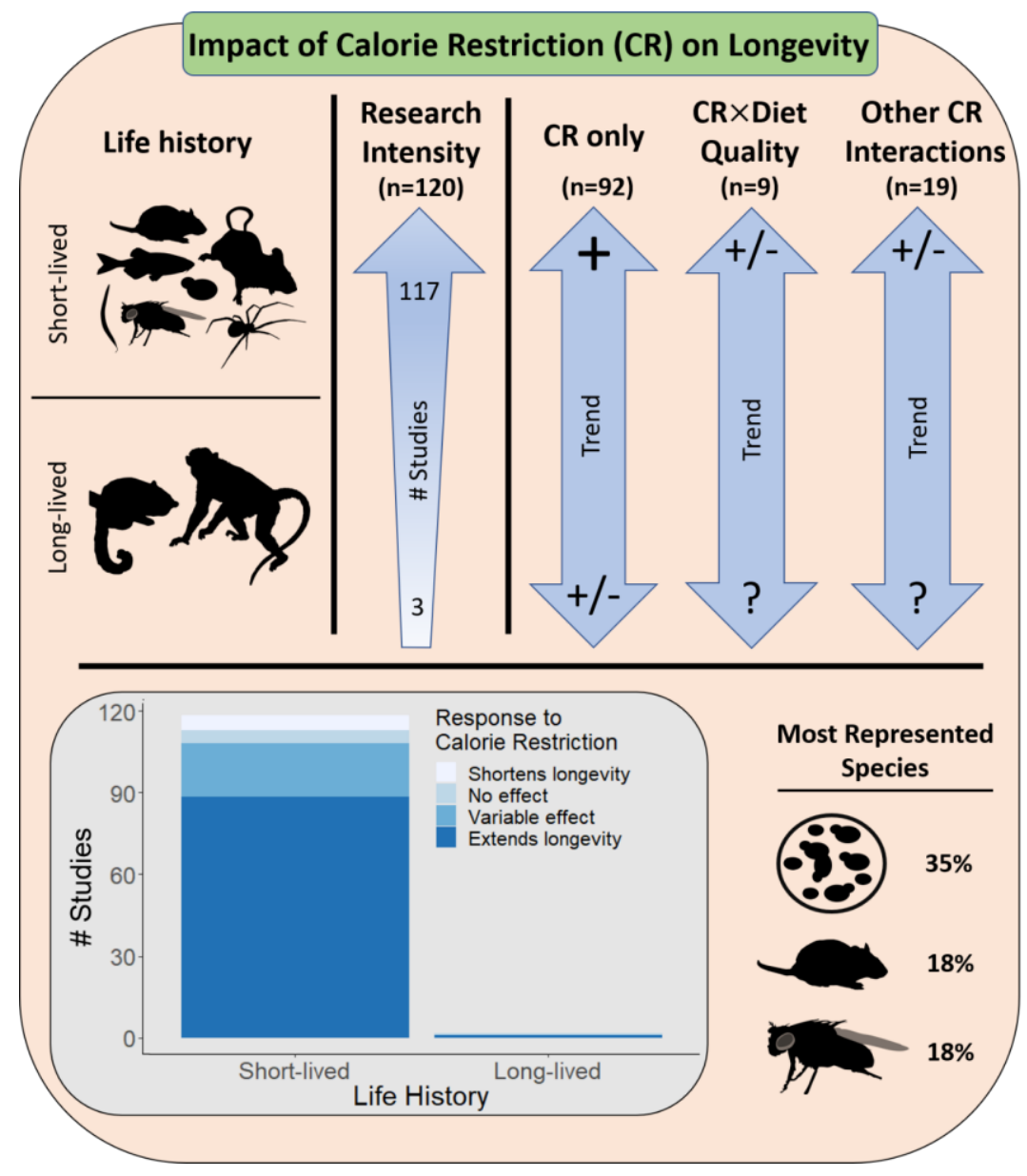

Figure 1. Summary results of a literature search of the impact of calorie restriction (CR) on longevity across 120 peer-reviewed studies between 1970 and 2020. Top panel: Life histories of examined species separated into short-lived and long-lived to identify differential impacts of CR impact on longevity. Research intensity corresponds to the number of studies focusing on short- and long-lived species. $C R$ only indicates the general effect of CR on longevity of studies where no other factor was investigated; $C R \times$ diet quality indicates the general effect of studies including $C R$ and diet quality interactions on longevity; Other CR interactions shows the general effect of CR studies that included factors other than $\operatorname{diet}$ (e.g. feeding frequency) on longevity. The general effects are ' + ' $=$ extends longevity; ' - ' $=$ shortens longevity; '+/-' = variable between and within studies (general effect is unclear); '?' = no studies. Silhouettes represent some of the organisms examined in this literature review (top to bottom and left to right): mouse (Mus musculus), zebrafish (Danio rerio), yeast (Saccharomyces cerevisiae), rat (Rattus norvegicus), nematode (Caenorhabditis elegans), fruit fly (Drosophila melanogaster), redback spider (Latrodectus hasselti), grey mouse lemur (Microcebus murinus) and rhesus macaque (Macaca mulatta). Bottom panel: Bar graph indicating the total number of studies in the literature review investigating the impact of CR on longevity in short- ( $<5$ years life expectancy) and long-lived species ( $>5$ years). Different colours in the bar graph indicate type of impact on longevity. The most represented species in the literature review are indicated to the right of the bar graph (as a percentage), correspond to yeasts, mice, and fruit flies (top to bottom).

To examine the current state-of-the-art and generality of CR theory, we identified and examined studies that focused on the impact of CR on longevity across species. Here, it is important to acknowledge the body of research in dietary restriction (DR), which focuses on the effects of dietary manipulations other than calorie intake, such as timing of feeding (e.g. ${ }^{24}$ ) or macro- and micronutrient manipulation (e.g. ${ }^{25}$ ). Thus, DR refers to an all-encompassing description for multiple forms of dietary interventions, with CR formally considered as a special case of $\mathrm{DR}^{26}$. Nonetheless, the terms $\mathrm{DR}$ and $\mathrm{CR}$ are used interchangeably by some authors ${ }^{27}$. In our literature review, we focus on $\mathrm{CR}$ 
research, thus ensuring studies with the aim of specifically manipulating calories. This is in line with CR theory, which predicts changes in longevity due to the ultimate effects of restricted food intake ${ }^{13}$. To that end, we compiled a literature search of peer-review publications from 1900 until March 2020 via Web of Science with the search terms "calorie" AND "restriction" AND "longevity" (see Supplementary Table 1). Of the 1,417 resulting publications, we excluded reviews and studies that did not directly test the impact of restricting calories on longevity (i.e., studies only investigating biomarkers of longevity $\left(\right.$ e.g. $\left.{ }^{28}\right)$, studies with genetic mutations or insertions $\left(\right.$ e.g. $\left.{ }^{29}\right)$ ). We also excluded studies only investigating the impact of calorie restriction mimetics $\left(e . g .{ }^{30}\right)$, as these works investigate compounds that mimic CR effects without actually restricting calorie intake itself. We were left with 120 original research studies, detailed in Supplementary Table 1.

Of the $120 \mathrm{CR}$ studies, the immense majority (97.5\%) focused on short-lived species (mean life expectancy $<5$ years), while studies on longer-lived species remain scarce $(2.5 \%$, Fig. 1$)$. The overall effect of CR on the longevity of short-lived species is significantly positive $\left(72.6 \% ; X_{1,117}^{2}=24.01, p<\right.$ 0.0001 ), with an increase in longevity ranging from $6.7 \%$ in the case of Rattus norvegicus to $10^{5}$ orders of magnitude in Saccharomyces cerevisiae (see Supplementary Table 1). Intriguingly, while two of the three studies on long-lived species in our literature review align with $\mathrm{CR}^{\prime}$ s predictions on expanded lifespan (e.g., rhesus macaque, Macaca mulatta ${ }^{31}$; grey mouse lemur, Microcebus murinus ${ }^{32}$ ), the third study does not show a significant extension in lifespan. The findings of the latter study are in direct odds with the results from another CR study on the same species, the rhesus macaque ${ }^{33}$ (Fig. 1). Likely reasons for this discrepancy include the lack of standardised protocols of nutritional demands ${ }^{34}$, or controls receiving an inadequate diet ${ }^{35}$. The importance of standardised protocols in CR studies has been raised in the past $\left(e . g .{ }^{36}\right)$ and reiterated in a recent review on experimental design limitations ${ }^{37}$. In the recent review, contradictory findings of the impact of $C R$ on longevity were attributable to methodological differences in feeding regimes, diet composition, age of onset, genetics, and sex. Indeed, this frequent -and still largely unattended- call for standardised protocols in CR suggests a need to formalise a framework for $\mathrm{CR}$ research, subsequent standardised protocols would then allow for between- and within-species comparison of the impact of CR on longevity.

Our review of the CR literature highlights the lack of interaction effects in CR studies, however including interaction effects is important to examine its consequences under realistic scenarios. For instance, the focus of the majority of the ageing literature has been on actuarial senescence (i.e., mortality risk changes with age after maturity, e.g. ${ }^{38}$ ) and not on reproductive senescence (but se $^{39,40}$ ). This is a significant knowledge gap, as classical senescence theories predict reproduction to decline as mortality risk increases with age ${ }^{41,42}$. However, recent work has shown that actuarial and reproductive senescence are often decoupled ${ }^{43}$, even though they are often assumed not to be ${ }^{38}$. A recent study ${ }^{43}$ suggests that key life history traits (i.e. organismal features that impact fitness, e.g. body size ${ }^{44}$ ) and ecology of the organism -including resource availability- may be crucial in shaping senescence outcomes. Thus, we argue that the impact of CR on senescence can only be satisfactorily identified in the context of both actuarial and reproductive senescence due to well-known trade-offs between survival and reproduction ${ }^{44}$. Of importance here too is the fact that different moments in the distribution of reproduction (e.g. frequency, intensity, duration) can be independent of investments in longevity in both animals ${ }^{45,46}$ and plants ${ }^{47}$, and so the mechanisms forcing an increase in mortality risk might be independent from those shaping age-specific reproduction.

The study of CR needs an explicit incorporation of life history theory to disentangle direct and indirect effects of resource availability. Indeed, CR reduces energy intake of individuals which, in long-lived species, life history theory predicts to result in a reduction or halting of reproduction ${ }^{44}$. Reduced reproduction, in turn, may free up resources for maintenance that then can increase longevity ${ }^{48}$. However, to disentangle direct and indirect effects of resource availability requires a greater understanding of the interaction of $\mathrm{CR}$ with other variables. In our literature search, only $23 \%$ $(n=28)$ of the $120 \mathrm{CR}$ studies focused on the interaction of $\mathrm{CR}$ with other variables such as diet quality ${ }^{49}$ or a stressor (e.g. oxidative stress ${ }^{50}$ ). Interestingly, the overall effect of $\mathrm{CR}$ and other factors on longevity was not significantly positive $\left(n=9 ; X_{1,28}^{2}=3.57, p=0.059\right)$, with the majority $(n=14)$ showing 
both positive and negative impacts (CR response $=$ 'variable', Supplementary Table 1$).$ Of the total number of studies that focus on the interaction of $C R$ and other variables, a third (32\%; $n=9)$ focused on the interaction of diet quality with CR (see Supplementary Table 1). None of the studies on the interaction of diet quality with CR showed positive impacts on longevity, with the majority revealing both positive and negative impacts within studies ( $n=6$; 'variable', Supplementary Table 1 ) or no impact at all ( $\mathrm{n}=2$; 'no effect', Supplementary Table 1). Our literature search highlights (1) the lack of studies investigating interaction effects in CR, (2) no support for the expected universally positive effects of CR on longevity in studies with interaction effects, and (3) a skewed focus on the interaction of diet quality and CR over other important factors such as feeding frequency or temperature. Therefore, we call for more research reproducing real-world scenarios and evolutionary pressures, such as experimental manipulations of feeding frequency or temporal autocorrelation of resource

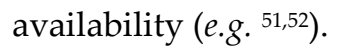

Theory on the impact of stochastic environments on life histories predicts that temporal variability in environmental quality strongly influences fitness ${ }^{53}$. Indeed, optimal phenotypes in fluctuating environments are expected to differ from optimal phenotypes in constant environments ${ }^{53}$, with the effect of serial correlation on fitness (i.e. increase or decrease in fitness through time) dependent on the life history of the organism (i.e. age specific survival and reproduction rates) ${ }^{53}$. Moving beyond constant conditions in experimental approaches in CR is especially key as variation in environmental quality causes variation in individuals' life history traits, such as age at maturity ${ }^{44}$. For example, some organisms mature earlier as environmental conditions become more favourable ${ }^{44}$ while others mature earlier when conditions are less favourable ${ }^{54}$. The documented vast range of life history responses to changes in environmental quality ${ }^{(5-58)}$ highlights the importance of interacting factors for determining longevity, and that the reported findings of $\mathrm{CR}$ in constant environments may not be consistent with those in fluctuating environments. Variable environments, in turn, play a crucial role in population dynamics by influencing survival and reproduction ${ }^{59}$. Furthermore, an increase in the variation in environmental quality has profound impacts on species through changes in habitat and structure of ecosystems $\mathbf{s}^{60,61}$. Examples include the change in synchrony with a species' food and habitat resources due to warm and/or dry years, as in Ediths' checkerspot butterfly (Euphydryas editha) and its host plant, the Torrey's blue eyed Mary (Collinsia torreyi), which results in population crashes and extinctions ${ }^{60,62}$. In our literature search, stochastic environments are much less represented and only investigated in short-lived species. Only two of the 120 studies, one study on Drosophila ${ }^{63}$ and another on medfly ${ }^{20}$, explicitly investigated CR impacts on senescence in stochastic environments. In these species, longevity was extended under a stochastic feeding regime when compared to constant environments, supporting CR predictions under real-world conditions. However, several environmental factors with interacting effects such as temperature and resource quality (below) are likely to influence how CR impacts organismal vitality in stochastic environments and may therefore be more accurate when examining CR impacts.

A key -yet often overlooked- environmental factor to consider in the context of $C R$ is temperature. For instance, mammals under $C R$ show reduced body temperature as a mediator of $C R$ on longevity ${ }^{64}$, and low body temperature can independently increase lifespan ${ }^{64}$. Likewise, in invertebrates, temperature can play a key role, particularly in expanding lifespan under cold conditions ${ }^{65}$. Furthermore, temperature can significantly affect nutrient assimilation efficiency. Plasman et al. ${ }^{66}$ show temperature differentially affects nutrient use in a lizard with higher temperatures increasing protein but decreasing lipid assimilation. So too can temperature impact the macronutrient requirement of organisms, with increasing temperatures resulting in the decline in the $\mathrm{N}$ and $\mathrm{P}$ content of whole organisms ${ }^{67}$. Consequently, understanding how resource $\times$ temperature interactions shape organismal vitality is key for projections of an organism's environmental niche space $^{68,69}$, as climatic models predict both factors to change ${ }^{70}$. Ultimately, how these interactions are impacted with a changing climate will dictate the quality of the full environmental niche space that the specific study species may experience. Thus, we argue that $\mathrm{CR}$ should be investigated in more ecologically realistic scenarios than in pristine, constant environments, as commonly done to date. 
Indeed, CR may become an increasing challenge in natural systems due to global climate change, given the uncertainty in the nature of future environments and each organism's response. From a human perspective, the impacts of climate change will not only influence future food production (quantity) $)^{71,72}$ but also the quality of the food that is produced ${ }^{72}$. As such, understanding CR in combination with factors such as diet composition, feeding regimes and temperature will be key when considering how CR impacts human health and well-being.

\section{Moving forward}

Addressing the consequences of $\mathrm{CR}$ in more realistic environments is a challenging but necessary prospect to advance ageing research. This challenge is especially apparent in species where the experimental logistics of determining relevant interactions are not feasible, such as in non-human primates and mice, where the required numbers for replicated designs are not feasible. However, a viable alternative is using study systems that can experimentally accommodate multiple effects to identify key CR interactions that impact senescence. Such systems would need to be easily maintained, allow for the necessary replication to ensure robust experimental designs, and preferably encompass short- and long-lived species.

Much CR research has focused on short-lived invertebrates like Drosophila ${ }^{63,73}$, including in the best of cases interaction effects ${ }^{63}$. Other promising short-lived systems that would allow for experiments investigating multiple interacting effects in high replication are yeast (Saccharomyces cerevisiae) and Caenorhhabditis elegans; these systems can be easily and quickly reared in the lab. In addition, we suggest two candidate systems to investigate key interactions that play a role in how CR impact senescence in long-lived species: Planarians and Hydra. Both systems are long-lived invertebrates (up to decades ${ }^{74-76}$ ) and can be lab-reared in high numbers while occupying little space $^{77,78}$. Interestingly, these long-lived systems have been studied to understand their regenerative properties and the apparent absence of ageing in certain species ${ }^{79,80}$. However, fewer studies have turned to Hydra as a system to explore the impact of CR and its interactions on longevity (e.g. ${ }^{81}$ ), with planarians yet to be utilised.

Long-lived invertebrate systems provide the opportunity to utilise predictions from life history theory to understand the impact of $\mathrm{CR}$ and its interaction effects on longevity. For example, selection pressures that increase lifespan result in a low mean and variance in adult mortality ${ }^{82}$. If factors that interact with $C R$ increase variation in adult mortality, this could negate the expected prolonged longevity under CR. Outcomes from such studies will then provide much needed insight into the role of CR on long-lived species and how life-history traits and whole populations respond to rapidly changing environmental conditions and resources driven by climate change.

Crucially, these insights from more realistic CR designs and on a broader range of taxa will contribute to the fundamental and translational understanding of human senescence. While we do not expect the mechanistic outcomes from the invertebrate studies to perfectly map to higher taxa, from a demographic and life history perspective, identifying the impacts of CR interaction effects on longevity encompassing short- and long-lived species will help us understand why some species senesce, but others do not' ${ }^{2}$. In particular, comparing long-lived and short-lived species within the same taxonomic group (e.g. rats live up to 5 years, while the naked mole-rat (Heterocephalus glaber) live for 30 years ${ }^{83}$ ) will provide a greater understanding of the confounding factors, due to varying evolutionary trajectories, that shape the relationships between $C R$ and longevity. CR has gained prime relevance in ageing research ${ }^{73,84}$, now more than ever in the light of climate change and its effects on securing resources ${ }^{61}$. However, only through standardised protocols applied to a wider variety of study systems that are not logistically constrained, can we address the heavily debated challenges currently facing $\mathrm{CR}$ research and finally test whether volunteering as a tribute in the Hunger Games does indeed postpone the onset of senescence and extends longevity.

Acknowledgments: This work was supported by a grant from the John Fell Fund (University of Oxford) awarded to R.S.G and A.A. 
Author contributions: J.A.D and R.S.G conceptualised the paper. J.A.D. and R.S.G. structured and drafted the article, A.A. contributed with input on CR mechanisms. All authors commented on and revised the paper. J.A.D conceived and developed Fig. 1, R.S.G. and A.A. contributed to Fig. 1.

Competing interests: The authors declare no competing interests.

\section{References}

1. Carone, G. et al. The Economic Impact of Ageing Populations in the Eu25 Member States. http://dx.doi.org/10.2139/ssrn.873872 (2005).

2. Jones, O. R. et al. Diversity of ageing across the tree of life. Nature 505, 169 (2014).

3. Harper, S. Economic and social implications of aging societies. Science 346, 587 (2014).

4. Ince Yenilmez, M. Economic and Social Consequences of Population Aging the Dilemmas and Opportunities in the Twenty-First Century. Appl. Res. Qual. Life 10, 735-752 (2015).

5. Bloom, D. E., Canning, D. \& Fink, G. Implications of population ageing for economic growth. Oxf. Rev. Econ. Policy 26, 583-612 (2010).

6. Vaupel, J. W. Biodemography of human ageing. Nature 464, 536-542 (2010).

7. Sierra, F., Hadley, E., Suzman, R. \& Hodes, R. Prospects for Life Span Extension. Annu. Rev. Med. 60, 457469 (2009).

8. Raffaelli, D. \& White, P. C. L. Chapter One - Ecosystems and Their Services in a Changing World: An Ecological Perspective. in Advances in Ecological Research (eds. Woodward, G. \& O'Gorman, E. J.) vol. 48 170 (Academic Press, 2013).

9. Boreux, V., Kushalappa, C. G., Vaast, P. \& Ghazoul, J. Interactive effects among ecosystem services and management practices on crop production: Pollination in coffee agroforestry systems. Proc. Natl. Acad. Sci. 110, 8387 (2013).

10. Baudisch, A. \& Vaupel, J. W. Getting to the Root of Aging. Science 338, 618 (2012).

11. Medvedev, Z. A. An attempt at a rational classification of theories of ageing. Biol. Rev. 65, 375-398 (1990).

12. Ross, G. R. T. Translation “Aristotle (350 BC) On Youth and Old Age, on Life and Death, on Breathing”. (2012).

13. Weindruch, R. \& Walford, R. L. The Retardation of Aging and Disease by Dietary Restriction. (Charles C Thomas, 1988).

14. Sohal, R. S. \& Weindruch, R. Oxidative Stress, Caloric Restriction, and Aging. Science 273, 59 (1996).

15. Fontana, L. \& Klein, S. Aging, Adiposity, and Calorie Restriction. JAMA 297, 986-994 (2007).

16. Cai, Y. \& Wei, Y.-H. Stress resistance and lifespan are increased in C. elegans but decreased in S. cerevisiae by mafr-1/maf1 deletion. Oncotarget 7, 10812-10826 (2016).

17. Lusseau, D. et al. The effects of graded levels of calorie restriction: IV. Non-linear change in behavioural phenotype of mice in response to short-term calorie restriction. Sci. Rep. 5, 13198 (2015).

18. McCay, C. M., Crowell, M. F. \& Maynard, L. A. The Effect of Retarded Growth Upon the Length of Life Span and Upon the Ultimate Body Size: One Figure. J. Nutr. 10, 63-79 (1935).

19. Masoro, E. J. Overview of caloric restriction and ageing. Mech. Ageing Dev. 126, 913-922 (2005).

20. Carey, J. R. et al. Stochastic dietary restriction using a Markov-chain feeding protocol elicits complex, life history response in medflies. Aging Cell 4, 31-39 (2005).

21. Colman, R. J. et al. Caloric restriction reduces age-related and all-cause mortality in rhesus monkeys. Nat. Commun. 5, 3557 (2014).

22. Bross, T. G., Rogina, B. \& Helfand, S. L. Behavioral, physical, and demographic changes in Drosophila populations through dietary restriction. Aging Cell 4, 309-317 (2005).

23. Mulvey, L., Sinclair, A. \& Selman, C. Lifespan Modulation in Mice and the Confounding Effects of Genetic Background. Spec. Issue Target. Ageing 41, 497-503 (2014).

24. Froy, O. \& Miskin, R. Effect of feeding regimens on circadian rhythms: implications for aging and longevity. Aging 2, 7-27 (2010).

25. Zimmerman, J. A., Malloy, V., Krajcik, R. \& Orentreich, N. Nutritional control of aging. Proc. 6th Int. Symp. Neurobiol. Neuroendocrinol. Aging 38, 47-52 (2003). 
26. Moatt, J. P., Savola, E., Regan, J. C., Nussey, D. H. \& Walling, C. A. Lifespan Extension Via Dietary Restriction: Time to Reconsider the Evolutionary Mechanisms? BioEssays 42, 1900241 (2020).

27. Richardson, A., Austad, S. N., Ikeno, Y., Unnikrishnan, A. \& McCarter, R. J. Significant life extension by ten percent dietary restriction. Ann. N. Y. Acad. Sci. 1363, 11-17 (2016).

28. Huffman, D. M. et al. Effect of exercise and calorie restriction on biomarkers of aging in mice. Am. J. Physiol.Regul. Integr. Comp. Physiol. 294, R1618-R1627 (2008).

29. Stenesen, D. et al. Adenosine Nucleotide Biosynthesis and AMPK Regulate Adult Life Span and Mediate the Longevity Benefit of Caloric Restriction in Flies. Cell Metab. 17, 101-112 (2013).

30. Calvert, S. et al. A network pharmacology approach reveals new candidate caloric restriction mimetics in C. elegans. Aging Cell 15, 256-266 (2016).

31. Colman, R. J. et al. Caloric Restriction Delays Disease Onset and Mortality in Rhesus Monkeys. Science 325, 201 (2009).

32. Pifferi, F. et al. Caloric restriction increases lifespan but affects brain integrity in grey mouse lemur primates. Commun. Biol. 1, 1-8 (2018).

33. Mattison, J. A. et al. Impact of caloric restriction on health and survival in rhesus monkeys from the NIA study. Nature 489, 318-321 (2012).

34. Cava, E. \& Fontana, L. Will calorie restriction work in humans? Aging 5, 507-514 (2013).

35. Lee, K. P. et al. Lifespan and reproduction in Drosophila: New insights from nutritional geometry. Proc. Natl. Acad. Sci. 105, 2498 (2008).

36. Troen, A. M. et al. Lifespan modification by glucose and methionine in Drosophila melanogaster fed a chemically defined diet. AGE 29, 29-39 (2007).

37. Vaughan, K. L. et al. Caloric Restriction Study Design Limitations in Rodent and Nonhuman Primate Studies. J. Gerontol. A. Biol. Sci. Med. Sci. 73, 48-53 (2018).

38. Jones, O. R. et al. Senescence rates are determined by ranking on the fast-slow life-history continuum. Ecol. Lett. 11, 664-673 (2008).

39. Sukhotin, A. A. \& Flyachinskaya, L. P. Aging reduces reproductive success in mussels Mytilus edulis. Mech. Ageing Dev. 130, 754-761 (2009).

40. Baudisch, A. \& Stott, I. A pace and shape perspective on fertility. Methods Ecol. Evol. 10, 1941-1951 (2019).

41. Medawar, P. B. An unsolved problem of biology. (H. K. Lewis, 1952).

42. Kirkwood, T. B. L. Evolution of ageing. Nature 270, 301-304 (1977).

43. Roper, M., Capdevila, P. \& Salguero-Gómez, R. Senescence: Still an Unsolved Problem of Biology. bioRxiv 739730 (2019) doi:10.1101/739730.

44. Stearns, S. C. The Evolution of Life Histories. (Oxford University Press, 1992).

45. Healy, K., Ezard, T. H. G., Jones, O. R., Salguero-Gómez, R. \& Buckley, Y. M. Animal life history is shaped by the pace of life and the distribution of age-specific mortality and reproduction. Nat. Ecol. Evol. 3, 12171224 (2019).

46. Paniw, M., Ozgul, A. \& Salguero-Gómez, R. Interactive life-history traits predict sensitivity of plants and animals to temporal autocorrelation. Ecol. Lett. 21, 275-286 (2018).

47. Salguero-Gómez, R. et al. Fast-slow continuum and reproductive strategies structure plant life-history variation worldwide. Proc. Natl. Acad. Sci. 113, 230-235 (2016).

48. Ng'oma, E., Perinchery, A. M. \& King, E. G. How to get the most bang for your buck: the evolution and physiology of nutrition-dependent resource allocation strategies. Proc. Biol. Sci. 284, 20170445 (2017).

49. Jensen, K., McClure, C., Priest, N. K. \& Hunt, J. Sex-specific effects of protein and carbohydrate intake on reproduction but not lifespan in Drosophila melanogaster. Aging Cell 14, 605-615 (2015).

50. Kaneko, G. et al. Calorie restriction-induced maternal longevity is transmitted to their daughters in a rotifer. Funct. Ecol. 25, 209-216 (2011).

51. Loureiro, F., Bissonette, J. A., Macdonald, D. W. \& Santos-Reis, M. Temporal Variation in the Availability of Mediterranean Food Resources: Do Badgers Meles meles Track Them? Wildl. Biol. 15, 197-206 (2009).

52. Grueter, C. C. et al. Long-Term Temporal and Spatial Dynamics of Food Availability for Endangered Mountain Gorillas in Volcanoes National Park, Rwanda. Am. J. Primatol. 75, 267-280 (2013). 
53. Tuljapurkar, S., Gaillard, J.-M. \& Coulson, T. From stochastic environments to life histories and back. Philos. Trans. R. Soc. Lond. B Biol. Sci. 364, 1499-1509 (2009).

54. Reznick, D., Bryant, M. \& Holmes, D. The Evolution of Senescence and Post-Reproductive Lifespan in Guppies (Poecilia reticulata). PLOS Biol. 4, e7 (2006).

55. Becker, F. S., Tolley, K. A., Measey, G. J. \& Altwegg, R. Extreme Climate-Induced Life-History Plasticity in an Amphibian. Am. Nat. 191, 250-258 (2018).

56. Coulson, T. et al. Modeling Effects of Environmental Change on Wolf Population Dynamics, Trait Evolution, and Life History. Science 334, 1275-1278 (2011).

57. Smallegange, I. M. Effects of paternal phenotype and environmental variability on age and size at maturity in a male dimorphic mite. Naturwissenschaften 98, 339-346 (2011).

58. Lindström, J. Early development and fitness in birds and mammals. Trends Ecol. Evol. 14, 343-348 (1999).

59. Coulson, T., Benton, T. G., Lundberg, P., Dall, S. R. X. \& Kendall, B. E. Putting evolutionary biology back in the ecological theatre: a demographic framework mapping genes to communities. Evol. Ecol. Res. 8, 11551171 (2006).

60. Parmesan, C. Ecological and evolutionary responses to recent climate change. Annu. Rev. Ecol. Evol. Syst. 37, 637-669 (2006).

61. IPCC. Climate Change 2014: Synthesis Report. in Contribution of Working Groups I, II and III to the Fifth Assessment Report of the Intergovernmental Panel on Climate Change (eds. Core Writing Team, Pachauri, R. K. \& Meyer, L. A.) 151 (IPCC, 2014).

62. Thomas, C. D., Singer, M. C. \& Boughton, D. A. Catastrophic Extinction of Population Sources in a Butterfly Metapopulation. Am. Nat. 148, 957-975 (1996).

63. Mair, W., Goymer, P., Pletcher, S. D. \& Partridge, L. Demography of Dietary Restriction and Death in Drosophila. Science 301, 1731-1733 (2003).

64. Carrillo, A. E. \& Flouris, A. D. Caloric restriction and longevity: Effects of reduced body temperature. Ageing Res. Rev. 10, 153-162 (2011).

65. Liu, R. K. \& Walford, R. L. The Effect of Lowered Body Temperature on Lifespan and Immune and NonImmune Processes. Gerontology 18, 363-388 (1972).

66. Plasman, M., McCue, M. D., Reynoso, V. H., Terblanche, J. S. \& Clusella-Trullas, S. Environmental temperature alters the overall digestive energetics and differentially affects dietary protein and lipid use in a lizard. J. Exp. Biol. 222, jeb194480 (2019).

67. Cross, W. F., Hood, J. M., Benstead, J. P., Huryn, A. D. \& Nelson, D. Interactions between temperature and nutrients across levels of ecological organization. Glob. Change Biol. 21, 1025-1040 (2015).

68. Kearney, M. R., Simpson, S. J., Raubenheimer, D. \& Kooijman, S. A. L. M. Balancing heat, water and nutrients under environmental change: a thermodynamic niche framework. Funct. Ecol. 27, 950-966 (2013).

69. Rho, M. S. \& Lee, K. P. Temperature-driven plasticity in nutrient use and preference in an ectotherm. Oecologia 185, 401-413 (2017).

70. Durant, J. M., Hjermann, D. O., Ottersen, G. \& Stenseth, N. C. Climate and the match or mismatch between predator requirements and resource availability. Clim. Res. 33, 271-283 (2007).

71. Porter, J. R. et al. Food security and food production systems. in Climate Change 2014: Impacts, Adaptation, and Vulnerability. Part A: Global and Sectoral Aspects. Contribution of Working Group II to the Fifth Assessment Report of the Intergovernmental Panel on Climate Change 485-533 (Cambridge University Press, 2014).

72. Scheelbeek, P. F. D. et al. Effect of environmental changes on vegetable and legume yields and nutritional quality. Proc. Natl. Acad. Sci. 115, 6804 (2018).

73. Liang, Y.et al. Calorie restriction is the most reasonable anti-ageing intervention: a meta-analysis of survival curves. Sci. Rep. 8, 5779 (2018).

74. Trouvé, S., Sasal, P., Jourdane, J., Renaud, F. \& Morand, S. The Evolution of Life-History Traits in Parasitic and Free-Living Platyhelminthes: A New Perspective. Oecologia 115, 370-378 (1998).

75. Elliott, S. A. \& Sánchez Alvarado, A. The history and enduring contributions of planarians to the study of animal regeneration. Wiley Interdiscip. Rev. Dev. Biol. 2, 301-326 (2013).

76. Tomczyk, S., Fischer, K., Austad, S. \& Galliot, B. Hydra, a powerful model for aging studies. Invertebr. Reprod. Dev. 59, 11-16 (2015). 
77. Sousa, N. de \& Adell, T. Maintenance of Schmidtea mediterranea in the Laboratory. Bio-Protoc. 8, e3040 (2018).

78. Lenhoff, H. Hydra: Research Methods. (Springer, 1983).

79. Boehm, A.-M., Rosenstiel, P. \& Bosch, T. C. G. Stem cells and aging from a quasi-immortal point of view. BioEssays 35, 994-1003 (2013).

80. Aboobaker, A. A. Planarian stem cells: a simple paradigm for regeneration. Trends Cell Biol. 21, 304-311 (2011).

81. Schaible, R., Ringelhan, F., Kramer, B. H. \& Miethe, T. Environmental challenges improve resource utilization for asexual reproduction and maintenance in hydra. Exp. Gerontol. 46, 794-802 (2011).

82. Stearns, S. C. Life history evolution: successes, limitations, and prospects. Naturwissenschaften 87, 476-486 (2000).

83. Buffenstein, R. The Naked Mole-Rat: A New Long-Living Model for Human Aging Research. J. Gerontol. Ser. A 60, 1369-1377 (2005).

84. Most, J., Tosti, V., Redman, L. M. \& Fontana, L. Calorie restriction in humans: An update. Nutr. Interv. Modul. Aging Age-Assoc. Dis. 39, 36-45 (2017).

(C) 2020 by the authors. Submitted for possible open access publication under the terms and conditions of the Creative Commons Attribution (CC BY) license (http://creativecommons.org/licenses/by/4.0/). 